\title{
The (Ab)use of Those with No Other Hope?
}

\author{
Ethical and Legal Safeguards for Recipients of \\ Experimental Procedures
}

\author{
SARA FOVARGUE
}

The idea that all members of a society have a duty to help promote scientific and medical advances has been the subject of debate, ${ }^{1}$ and it has recently been suggested that patients should be able to participate in "research that may be beyond the bounds of current clinical research practice" and that "even when greater-than-usual risks may be present, such research can be both ethically and scientifically justified." 2 This is partly based on the argument that all of us are obliged to participate in research and that where there is "some reasonable likelihood

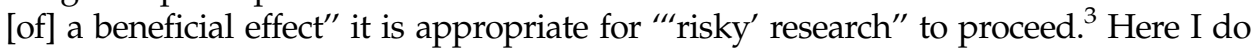
not dispute this contention but draw attention to some of the issues that are raised by involving those with no other hope in experimental procedures. These are important because of the position-desperately ill with no alternatives-such patients are in. The UK's Nuffield Council on Bioethics has recognized that medical progress has sometimes been made at the "expense" of those who first received a new procedure, and that "[i]n some cases, it has been cruel to offer a possible life-saving procedure that resulted in a long drawn-out painful death, instead of a relatively peaceful end. The offer of such a procedure in itself puts pressure on patients to accept-and may distort judgement." ${ }^{\prime 4}$ I am not arguing that there should be a blanket ban on involving those with no other hope in experimental procedures; rather, I argue that, if they are to be involved, then they must be legally protected and that the use of such procedures is scientifically justified and ethically reviewed.

In this article I examine international and national ethical and professional guidance on offering experimental procedures to those with no other hope and explore the common law on experimental procedures in England and Wales. The issues highlighted here are not jurisdiction specific; rather, the regulatory framework in England and Wales is used as a lens through which to explore and highlight the protection offered to those with no other hope. In so doing I question whether the existing regulatory structures appropriately protect those with no other hope and draw on ethical guidance from New Zealand that suggests ways to proceed. Care must be taken with involving such patients in experimental procedures because they may be particularly vulnerable and open to exploitation, even if this is not the deliberate or conscious aim of those seeking to perform the procedure for the first time. Despite this risk, selection issues in this context have rarely been considered. This may be because their involvement has been assumed

This article is dedicated to Professor David Price, who was an inspirational and supportive academic, colleague, and mentor. 


\section{Sara Fovargue}

to be unproblematic, because if the patients are competent, they can give their informed consent to participate and are also able to make other "risky" choices, such as refusing life-saving treatment. ${ }^{5}$ However, justice may limit the harms to which a person can be subjected, ${ }^{6}$ and if it is acceptable to involve those with no other hope in experimental procedures, then it is imperative that they are appropriately protected. At the same time, their ability to access opportunities to prolong or save their lives should not be unnecessarily impeded.

\section{Definitional Dilemmas}

One of the problems in this field is that it is unclear what an experimental procedure is. Terms such as "research," "experiment," "treatment," "procedure," "therapy," and "practice" and the adjectives "new," "innovative," or "experimental" are used interchangeably and inconsistently in existing literature, ${ }^{7}$ possibly because some of these terms are not legally defined. For example, the UK's Royal College of Physicians (RCP) discusses "medical research," "medical practice," and "innovative medical practice" in the same paragraph, followed by "innovative therapy" and then the specific provisions relating to "new interventional procedures," which are also (confusingly) termed "an experiment." ${ }^{\prime 8}$ Research in the form of a clinical trial is statutorily defined in England and Wales, ${ }^{9}$ as is "intrusive research" involving those without legal capacity. ${ }^{10}$ Outside of these situations, the law does not assist, so "therapy," "treatment," "experiment(al)," "new," or "innovative" are not defined. A number of bodies, including the RCP, Department of Health (DH), and the General Medical Council (GMC), define "research," but these definitions are not legally binding, although they are professionally important. ${ }^{11}$ The $\mathrm{DH}$ has, however, defined an "interventional procedure" as "one used for diagnosis or treatment that involves incision, puncture, entry into a body cavity, electromagnetic or acoustic energy," and "[a]n interventional procedure should be considered new if a doctor no longer in a training post is using it for the first time in his or her NHS clinical practice." ${ }^{\prime 12}$

This definition of a new interventional procedure (NIP) may encompass some of the activities that are elsewhere called experimental (or novel or innovative) procedures (or therapies or treatments or practices). For example, in New Zealand "innovative practice" involves "the provision of a clinical intervention (diagnostic, therapeutic or prophylactic), be it a therapeutic drug, medical device or clinical procedure, that is untested, unproven or not in common use and therefore poses its own unique set of characteristics and issues."13 The goal is to "provide some immediate treatment in relation to an individual consumer or consumer group concerned" and performing it "may be considered to be a planned deviation from current accepted practice of a New Zealand body of health professionals involving an untested or unproven clinical intervention intended to be used on an ongoing basis." ${ }^{\prime 14}$ Not every deviation from accepted practice should be deemed as innovative, and professional judgment, preferably following consultation with relevant experts, will determine whether something is an innovative practice. ${ }^{15}$ If it is unclear whether an intervention is an innovative practice, then an ethics committee should be consulted. ${ }^{16}$

The lack of clear legal definitions immediately places potential recipients in a regulatory grey area, as it may be unclear whether a procedure is (or should be) declared research, a NIP, or an experimental procedure. Their legal protection is 
thus questionable. There is undoubtedly a fine line between experimental procedures/therapies/treatments and research, and although it may be theoretically and practically difficult to distinguish between these activities (and it may not ethically matter what something is called) ${ }^{17}$ it is necessary to do so, as different legal regulatory schemes apply to each. Indeed, clinical trials are statutorily regulated in England and Wales; ${ }^{18}$ medical research not included within this scheme is regulated by a combination of the common law, guidance from professional bodies, ${ }^{19}$ and any requirements imposed by ethics committees, ${ }^{20}$ and NIPs are regulated by nonlegally binding guidelines from the National Institute for Health and Clinical Excellence (NICE). ${ }^{21}$ Experimental procedures may fall within one of these regulatory schemes; otherwise the common law will apply.

To be clear, here I use "experimental procedure" to mean "novel and unvalidated . . . an 'experiment' is a procedure adopted on the chance of its succeeding." 22 It "consists in the performance of a new or non-standard intervention as all or part of a therapeutic activity and not as part of a formal research project." ${ }^{23} \mathrm{I}$ employ this term to highlight the fact that using it is an experiment (a test), as it has not previously been clinically used, and it is not known what will happen when it is used in or performed on a human. It cannot yet be called "treatment" or "therapy" because both of these terms suggest that it has proven benefit, and this is not so where the relevant activity has not been clinically tried. ${ }^{24}$ The term "procedure" is also appropriately neutral and covers a wide range of activities.

\section{Identifying the Problem}

Involving those with no other hope in experimental procedures raises a number of questions, ${ }^{25}$ but here my focus is on how experimental procedures are regulated and whether the particular situation of those with this health status is recognized and additional protection thereby afforded to them. Such patients may be offered experimental procedures because there are no other alternatives for them, and they may accept because of the chance of benefit, even though it is more likely that the results will benefit others. It is understandable why they would want to take this chance, but what society asks of people "reflect[s] how we as a society value individuals and what we consider to be fair and reasonable burdens." ${ }^{26}$ Therefore, it is important to consider what we ask of those with no other hope. Three questions underpin my concerns: Does asking those with no other hope to receive an experimental procedure untested on humans imply that their lives are less valuable, or does it indicate that their lives remain of value and so we strive to prolong them? Can (and should) limits be placed on who and what experimenters ask, because the very act of asking makes the other person feel under some duty or responsibility to agree? At the same time, should those with no other hope be denied the smallest, remotest chance of benefit, or is it discriminatory and unethical to automatically exclude them from experimental procedures?

Being the first recipient of an experimental procedure can be advantageous; for example, the first allotransplant patients were viewed as "esteemed and heroic companions in a perilous but promising group endeavour that makes 'front-line' kindred of all participants." ${ }^{27}$ As such they were given special considerations, were involved in their treatment almost as professional medical peers, and were provided with technical information about their disorders and the experiments they were 


\section{Sara Fovargue}

participating in; in addition, the shared "awareness of impending death" further intensified the relationship between the medical team and their patients. ${ }^{28}$ The health professionals caring for those with no other hope may well be frustrated by the lack of options for their patient and thus may, understandably, want to try an experimental procedure when there is no other alternative. This frustration may not be confined to these professionals, and some patients with no other hope enroll in clinical trials because there is no standard treatment for them. ${ }^{29}$ Others have sought to characterize experimental procedures as treatment because they represent their only hope of improving or extending their life. ${ }^{30}$ Participation is thus not "an unqualified sacrifice" but "a potentially risky opportunity," 31 and involvement can provide unexpected benefits, such as uncovering previously unknown abnormalities. ${ }^{32}$ Conversely, providing experimental therapies or drugs outside a clinical trial, for example, raises safety issues, may minimize the number of participants subsequently available for trials, and undermines the clinical trial process. ${ }^{33}$

Concern over the possible (ab)use of those with no other hope is not hypothetical, because such patients received the first artificial cardiac devices, were involved in initial allotransplants, and took part in the nongenetically engineered xenotransplants performed in the 1960s. ${ }^{34}$ It has recently been suggested that experimental cancer drugs and stem cell "therapies" should involve those who are terminally ill, and that only patients at risk of dying and with no alternative treatment available should be included in a genetically engineered xenotransplant trial. ${ }^{36}$ Notably, in 2006 the English High Court sanctioned a patient in a persistent vegetative state receiving experimental "treatment." 37 Thus, it is important to consider the extent to which such patients are currently protected.

\section{Ethical Guidance on Providing Experimental Procedures}

Unless it is assumed that international ethical guidance on medical research also applies to experimental procedures, there is no specific international ethical guidance on providing these procedures. If this research guidance does apply then, for example, the Declaration of Helsinki (the Declaration) ${ }^{38}$ and the Council for International Organizations of Medical Sciences (CIOMS) and World Health Organization (WHO) guidelines ${ }^{39}$ would require a protocol that is ethically reviewed and approved before the procedure is performed, and it must include a risk-benefit analysis in which the latter outweighs the former. ${ }^{40}$ Informed consent is also required prior to participation. ${ }^{41}$ These preconditions aim to ensure that the protocol reflects the principles of respect for persons and autonomy, beneficence, nonmaleficence, and justice, which are specifically noted in the CIOMS and WHO research guidelines, ${ }^{42}$ and implied in the Declaration. ${ }^{43}$ Alongside these more general provisions regarding research and (presumably) experimental procedures, the Declaration also states that

[i]n the treatment of a patient, where proven interventions do not exist or have been ineffective, the physician, after seeking expert advice, with informed consent from the patient or a legally authorized representative, may use an unproven intervention if in the physician's judgement it offers hope of saving life, re-establishing health or alleviating suffering. Where possible, this intervention should be made the object of research, designed to evaluate its safety and efficacy. ${ }^{44}$ 
This vague and wide-ranging justification appears to support the use of experimental procedures for those with no other hope, but there is no indication as to how, or whether, the doctor's judgment is assessed other than via the advice of unspecified "experts." Although this provision sits under the heading "Additional Principles for Medical Research Combined with Medical Care," it permits unproven interventions (experimental procedures in my terminology) to be performed outside of a research project. If this occurs, it is unclear whether a protocol and/or ethics committee approval is required, although the first provision of the Declaration states that " $t]$ he Declaration is intended to be read as a whole and each of its constituent paragraphs should not be applied without consideration of all other relevant paragraphs." ${ }^{\prime 45}$ With regard to any specific issues raised by recruiting and involving those with no other hope, the Declaration is silent.

In contrast, the CIOMS and WHO research guidelines state that "[s]pecial justification is required for inviting vulnerable individuals to serve as research subjects and, if they are selected, the means of protecting their rights and welfare must be strictly applied." "Vulnerable" persons include "patients with incurable disease," and "[p]atients who have serious, potentially disabling or life-threatening diseases are highly vulnerable." The commentary to Guideline 13 notes that "[p]hysicians sometimes treat such patients with drugs or other therapies not yet licensed for general availability because studies designed to establish their safety and efficacy have not yet been completed" and declares that this "compassionate use' ... not properly regarded as research" contributes to "ongoing research into the safety and efficacy of the interventions used." ${ }^{\prime 7}$ Compassionate use is regarded as compatible with paragraph C32 of the 2002 version of the Declaration of Helsinki, which was similar to and preceded C35, noted previously. With regard to selecting (research) participants, the CIOMS and WHO guidelines state that care should be taken to ensure that the benefits and burdens are equitably distributed, and the exclusion of groups or communities who might benefit from participation must be justified. ${ }^{48}$ In particular, "[m]embers of vulnerable groups . . . have the same entitlement to access to the benefits of investigational interventions that show promise of therapeutic benefit as persons not considered vulnerable, particularly when no superior or equivalent approaches to therapy are available." ${ }^{\prime 4}$ Those with no other hope should thus have the opportunity to benefit from being involved (in research), provided the patient or his or her legal representative consents. ${ }^{50}$ As vulnerable groups should have the chance to benefit from research, and the "compassionate use" of drugs or therapies "not properly regarded as research" is endorsed, it appears that experimental procedures can be performed on those with no other hope under the CIOMS and WHO ethical research guidelines.

Although these international guidelines are important, they are just guidelinesguides to practice - and it is not mandatory to follow them. In England and Wales, the GMC's guidelines are the most important professional ethical guidance for doctors because failure to comply with them may result in disciplinary action. ${ }^{51}$ The GMC's research guidelines do not apply to "innovative treatments designed to benefit individual patients, ${ }^{\prime \prime 2}$ but its guidance on consent and confidentiality do. ${ }^{53}$ The consent guidance requires that patients are given the information they "want or need" on whether what is proposed "is part of a research programme or is an innovative treatment designed specifically for their benefit," and that they are told "how the proposed treatment differs from the usual methods, why it is being offered, and if there are any additional risks or uncertainties." ${ }^{24}$ The GMC offers no further 


\section{Sara Fovargue}

guidance on providing experimental, new, or innovative procedures, treatments, or therapies and does not require a protocol for "innovative treatment" or for it to be approved by an ethics committee. Similarly, in the case of "innovative therapy," the RCP's guidelines state that clinicians should be prepared to "both ethically and scientifically" justify its use if challenged, ${ }^{55}$ but the RCP does not require a protocol or ethics committee approval. Rather, reliance is placed on individual professional judgment as to the suitability of and justification for its use.

In comparison, the New Zealand Ministry of Health's standards for ethics committees do apply to innovative practices. ${ }^{56} \mathrm{~A}$ protocol is thus required that must be ethically reviewed, ${ }^{57}$ and the ethics committee must be satisfied with the information available for a patient to make an informed decision, that the purpose is to treat a condition of a particular patient or group, that there are "appropriate safeguards" for independent clinical assessment so that if necessary the patient(s) can change to standard treatment instead, and there are "appropriate evaluative mechanisms" to assess the effectiveness of the practice. ${ }^{58}$ The standards set out seventeen pieces of information to be considered by the committee, including the purpose, justification, and clinical indications for using the innovative practice; how recipients are to be selected; the information they will receive; and how informed consent will be obtained. ${ }^{59}$ Although these provisions are more specific and extensive than those set out in professional guidance in England and Wales, in neither jurisdiction is there a sense that providing an experimental procedure to those with no other hope raises specific legal or ethical problems.

\section{The Law on Providing New Interventional Procedures and Experimental Procedures in England and Wales}

Experimental procedures are neither defined nor statutorily regulated, but they may be regulated under HSC 2003/011 if they fall within the definition of a NIP. If so, a practitioner who wants to perform a NIP should seek approval from his or her NHS trust's clinical governance committee and should notify the Interventional Procedures Programme at the NICE if the procedure is not already listed by the NICE. ${ }^{60}$ If it is not listed, then once the NICE is notified of a NIP, a brief overview of the evidence of its safety and efficacy will be prepared, specialist advisors will be consulted, a NICE advisory committee will decide whether to issue guidance on it or to ask for more information, and the public will be consulted on the guidance. ${ }^{61}$ A NIP should only be approved for use if (1) the doctor has met externally set standards of training; (2) patients are made aware of the procedure's special status and the lack of experience of its use, are informed of the "anticipated benefits and possible adverse effects" of the procedure and any alternatives-including no treatment-and understand "that the procedure's safety and efficacy is uncertain," as part of the consent process; and (3) the proposed arrangements for clinical audit are sound and capture data on clinical outcomes that can be used to review its continued use. ${ }^{62}$ However,

in rare circumstances, where no other treatment options exist, there may be a need to use a new procedure in a clinical emergency so as not to place a patient at serious risk. If a doctor has performed a new interventional procedure in such circumstances he/she must inform the Clinical 
Governance Committee within 72 hours. The Committee will consider approval of the procedure for future use. ${ }^{63}$

A NIP can thus be clinically used in this situation and without ethical or other review or notification.

There are five important points to note about this circular. First, it was due for review on November 12, 2010, but nothing further has been published. ${ }^{64}$ Its status is thus unclear, and if it no longer applies, an additional gap in the regulation of NIPs exists. Second, if the NICE decides that what is proposed is a NIP under HSC 2003/011 then it does not have to be ethically reviewed, and the relevant doctor or experimenter will determine who receives it. Third, the circular does not apply to a procedure that meets the definition of a NIP but that is being used within an ethics committee-approved trial. ${ }^{65}$ Thus, some NIPs will be reviewed by an ethics committee and others will not, including those performed "where no other treatment options exist." ${ }^{66}$ Fourth, the legal status of health service circulars is unclear. These circulars are "If]ormal communications, primarily to NHS chief executives, which usually contain a requirement for significant or urgent specific action. Many are quasi-legislative." ${ }^{\prime 67}$ The legal status of HSC 2003/011 is unclear, and there are no sanctions included within it for noncompliance. Similarly, NICE guidance itself is not legally binding, although there is an expectation that professionals will take it "fully into account" when exercising clinical judgment. ${ }^{68}$ It does not, however, "override the individual responsibility of health professionals to make appropriate decisions according to the circumstances of the individual patient in consultation with the patient"; thus, "[i]f, having considered that guidance, a health professional considers that the treatment or procedure would be the appropriate option in a given case, there is no legal bar on the professional recommending the treatment or on the NHS funding it." ${ }^{69}$ If the doctor or experimenter does not follow NICE guidance, then he or she may rely on the common law to establish that the decision to offer a patient a NIP was justified. This is the position if an experimental procedure is deemed not to fall within the definition of a NIP in HSC 2003/011. The Bolam test obviously cannot be used to support such a decision, because, when the procedure is experimental, it is unlikely that there will be a responsible body of medical opinion to support its use, ${ }^{70}$ but "where the patient's condition is very serious and the standard treatment is ineffective, a doctor will be justified in taking greater risks in an attempt to provide some effective treatment." 71

Finally, the provisions in HSC 2003/011 regarding the consent process suggest that recipients of NIPs must be legally competent. ${ }^{72}$ Applying the common law on consent to treatment to NIPs and experimental procedures, a competent person can (presumably) consent to such provided they are informed "in broad terms of the nature of the procedure which is intended." 73 The House of Lords has recognized that it is possible to consent to procedures such as surgery, even though this will necessarily cause bodily harm "on the upper side of the critical level," provided it is "performed in accordance with good medical practice and with the consent of the patient." ${ }^{\prime 74}$ In the research context this would mean that ethics committee approval was sought, and the same might also apply to NIPs and experimental procedures, but "good medical practice" in this area is legally unclear and untested, and, of course, NICE guidance on NIPs does not require ethical review. Whether those who lack the legal capacity to consent can also receive NIPs and experimental 


\section{Sara Fovargue}

procedures is similarly uncertain, but, as I now discuss, common law suggests that incapacity is no bar.

\section{Providing an Experimental Procedure in Practice}

Judicial dicta on providing experimental procedures in England and Wales are limited, but in Simms v. Simms ${ }^{75}$ JS and JA suffered from probable variant Creutzfeldt-Jakob disease (vCJD), and, at that time, there were no recognized effective drugs to halt neurological deterioration or prolong life. Research on nonhuman animals indicated that pentosan polysulphate (PPS) inhibited the abnormal prion proteins that caused the neurological damage, but the effect of PPS on humans at an advanced stage of vCJD (such as JS and JA) was unknown. Because of their illnesses, JS and JA were unable to consent to PPS, and so their parents sought a declaration that it would be lawful to provide it to them in their best interests. Three of the four experts who gave evidence concluded that providing PPS was in JS and JA's best interests. Dame Elizabeth Butler-Sloss P. stated that JS and JA lacked the capacity to decide for themselves and that "[w]here there is no alternative treatment available and the disease is progressive and fatal, it seems . . . to be reasonable to consider experimental treatment with unknown benefits and risks, but without significant risks of increased suffering to the patient, in cases where there is some chance of benefit to the patient."76 Furthermore, "[a] patient who is not able to consent to pioneering treatment ought not to be deprived of the chance in circumstances where he would have been likely to consent if he had been competent."77 Participation in "pioneering treatment" thus appears open to all, but Dame Butler-Sloss was "unclear" whether providing PPS to those without capacity would fall within the Bolam test. ${ }^{78}$ Nevertheless, she noted that it could not be "allowed to inhibit medical progress," and "if one waited for the 'Bolam test' to be complied with to its fullest extent, no innovative work . . . would ever be attempted."79 Thus, although there was not a professional body of opinion endorsing PPS treatment, there was "evidence from responsible medical opinion which does not reject the research," 80 so "it would not in itself be irresponsible or unethical to give the treatment to these patients." 81 There was "a responsible body of relevant professional opinion which supports this innovative treatment," subject to the seriousness of the risks involved and the degree of benefit that might be achieved, and the reasonableness of the risks should be discussed within the patients' best interests. ${ }^{82}$ The benefits of PPS were "less tangible and more difficult to assess," 83 and there could be no obvious benefit or no benefit at all; however, "it [could not] be said that in principle this is treatment which is clearly futile or that it would not, in suitable cases be proper to give the PPS treatment to those suffering from prion disease, and I am therefore satisfied that the proposed PPS treatment complies with the 'Bolam test."' 84

The decision in Simms $v$. Simms is important because it involves what Dame Butler-Sloss variously termed "experimental," "pioneering," and "innovative" "treatment" that had not previously been used on humans, and the intended recipients were in a terminal, no-other-hope situation. The precedent value of this case is unclear because " $[\mathrm{t}]$ he extreme nature of the circumstances alone means that similar facts are likely to arise only rarely" ${ }^{\prime 85}$; nevertheless, it indicates that a court can approve the use of an experimental procedure ("treatment") for those 
with no other hope outside a clinical trial and without its attendant support mechanisms, including ethical review.

\section{Conclusion}

Experimental procedures must be ethically performed, and involving someone who is dying "anyway" does not excuse unethical investigations. The issues raised here must be considered so that the most seriously ill and understandably desperate patients are not used as mere tools for society's apparent benefit. In some situations in which experimental procedures are being considered for clinical use, additional guidelines may be drafted that might address recipient selection and some of the concerns expressed here. But this is not always the case, and such guidelines are not mandatory. For example, a genetically engineered solid organ xenotransplant can be clinically performed in England and Wales outside the context of a clinical trial or research approved by a research ethics committee, as "experimental medicine"; in this situation, approval is only required from the trust's clinical governance committee under HSC 2003/011. ${ }^{86}$ That this is the case is extraordinarily worrying, given the risks of this developing biotechnology. ${ }^{87} \mathrm{I}$ am not arguing that those with no other hope should not be able to receive experimental procedures (or participate in research); rather, I argue that their involvement must be carefully considered and their safety and health (however limited) protected. As such, a legal definition of an experimental procedure is required, and such procedures, as well as NIPs as defined by the DH in HSC 2003/011, must be performed under a protocol that is subject to ethical review. Thus, the New Zealand system relating to innovative practices should be considered. Without such it is hard to see how help and harm can be balanced in the light of hope, but this is necessary to ensure that exploitation of those with no other hope is minimized.

\section{Notes}

1. Caplan AL. Is there a duty to serve as a subject in biomedical research? IRB: Ethics and Human Research 1984;6:1-5; Harris J. Scientific research is a moral duty. Journal of Medical Ethics 2005; 31:242-8; Brassington I. Defending the duty to research? Bioethics 2011;25:21-6.

2. Chan S, Zee Y-K, Jackson G, Harris J. "Risky" research and participants' interests: The ethics of phase 2C clinical trials. Clinical Ethics 2011;6:91-6, at 91-2.

3. See note 2, Chan et al. 2011, at 91-2.

4. Nuffield Council on Bioethics. Animal-to-Human Transplants-The Ethics of Xenotransplantation. London: Nuffield Council on Bioethics; 1996, at para. 7.7. See also para. 7.4.

5. See, e.g., Re T (Adult: Refusal of Treatment) [1992] 4 All ER 649, CA.

6. Kong WM. Legitimate requests and indecent proposals: Matters of justice in the ethical assessment of phase I trials involving competent patients. Journal of Medical Ethics 2005;31:205-8.

7. Mason JK, Laurie GT. Mason and McCall Smith's Law and Medical Ethics. Oxford: Oxford University Press; 2011, at 612-3; Plomer A. The Law and Ethics of Medical Research: International Bioethics and Human Rights. London: Cavendish; 2005, at 47-8.

8. Royal College of Physicians (RCP). Guidelines on the Practice of Ethics Committees in Medical Research Involving Human Subjects. 4th ed. London: Royal College of Physicians; 2007, at para. 3.4-3.7 respectively.

9. Regulation 2(1), The Medicines for Human Use (Clinical Trials) Regulations 2004, SI 2004 No. 1031 as amended.

10. Section 30 Mental Capacity Act 2005.

11. See note 8, RCP 2007, at para. 3.1-3.2 and 3.4; Department of Health (DH). Research Governance Framework for Health and Social Care. 2nd ed. London: DH; 2005 Annex 2008, at para. 1.10; General Medical Council (GMC). Good Practice in Research. London: GMC; 2010, at para. 1, respectively. 


\section{Sara Fovargue}

12. HSC 2003/011. The Interventional Procedures Programme-Working with the National Institute for Clinical Excellence to Promote Safe Clinical Innovation, at para. 16, emphasis in original.

13. Ministry of Health (MH). Operational Standard for Ethics Committees. Wellington: MH; 2006, at para. 116.

14. See note 13, MH 2006, at para. 118 and 121 respectively

15. See note 13, MH 2006, at para. 120 and 125 respectively.

16. See note 13, MH 2006, at para. 125-126.

17. See note 2, Chan et al. 2011, at 92 .

18. See note 9, The Medicine for Human Use (Clinical Trials) Regulations 2004.

19. See note 8, RCP 2007; see note 11, GMC 2010.

20. For a helpful overview, see Stauch M, Wheat K, with Tingle J. Text, Cases and Materials on Medical Law and Ethics. 4th ed. London: Routledge; 2011, at 487-98.

21. National Institute for Health and Clinical Excellence (NICE). The Legal Implications of NICE Guidance; 2004; available at http://www.nice.org.uk/media/8BD/2B/Legal_context_nice_guidance. pdf (last accessed 23 Nov 2012).

22. See note 8 , RCP 2007, at para. 3.5, emphasis supplied.

23. Nicholson RH, ed. Medical Research with Children: Ethics, Law, and Practice-The Report of an Institute of Medical Ethics Working Group on the Ethics of Clinical Research Investigations on Children. Oxford: Oxford University Press; 1986, at 36.

24. McLean S. Gene therapy-Cure or challenge? In Freeman M, Lewis A, eds. Law and Medicine Current Legal Issues. Vol. 3. Oxford: Oxford University Press; 2000, at 205-22.

25. These questions include issues regarding competence to consent and who makes selection decisions, as is discussed in Fovargue S. Xenotransplantation and Risk: Developing a Developing Biotechnology. Cambridge: Cambridge University Press; 2012, at chap. 3 and 5.

26. See note 6, Kong 2005, at 207.

27. Fox RC, Swazey JP. The Courage to Fail-A Social View of Organ Transplants and Dialysis. Edison, New Jersey: Transaction; 2002, at 105.

28. Christopherson LK, Lunde DT. The selection of cardiac transplant recipients and their subsequent psychological adjustment. Seminars in Psychiatry 1971;3:36-45, at 40.

29. Boseley S. Human guinea pigs lend their courage to a golden era of cancer research. The Guardian 2011 Aug 22.

30. Hughes V. When patients march in. Nature Biotechnology 2010;28:1145-8. See also, e.g., the lobbying that occurred around the use of Herceptin, as evidenced in articles in The Guardian during 2005-2006.

31. Fox M. Research bodies: Feminist perspectives on clinical research. In Sheldon S, Thomson M, eds. Feminist Perspectives in Health Care Law. London: Cavendish; 1998:115-34, at 123.

32. Orme NM, Fletcher JG, Siddiki HA, Harmsen WS, O'Byrne MM, Port JD, et al. Incidental findings in imaging research: Evaluating incidence, benefit, and burden. Archives of Internal Medicine 2010;170:1525-32.

33. Chahal M. Off-trial access to experimental cancer agents for the terminally ill: Balancing the needs of individuals and society. Journal of Medical Ethics 2010;36:367-70.

34. McLean SAM, Williamson L. Xenotransplantation: Law and Ethics. Aldershot: Ashgate; 2005, at 29-30; Hardy JD, Chavez CM. The first heart transplant in man-Developmental animal investigations with analysis of the 1964 case in the light of current clinical experience. American Journal of Cardiology 1968;22:772-81; Reemtsma K, McCracken BH, Schlegel JU, Pearl MA, Pearce CW, DeWitt CW, et al. Renal heterotransplantation in man. Annals of Surgery 1964;160:384-408; Starzl TE, Marchioro TL, Peters GN, Kirkpatrick CH, Wilson WEC, Porter KA, et al. Renal heterotransplantation from baboon to man: Experiences with 6 cases. Transplantation 1964;2:752-76.

35. See note 33, Chahal 2010, at 367; Gray L. Dying can aid stem cell research. The Scotsman 2005 Dec 27.

36. Welin S. Starting clinical trials of xenotransplantation-Reflections on the ethics of the early phase. Journal of Medical Ethics 2000;26:231.

37. B NHS Trust v. J [2006] EWHC 3152.

38. World Medical Association (WMA). Declaration of Helsinki, Ethical Principles for Medical Research Involving Human Subjects, adopted by the 18th WMA, Helsinki, Finland, June 1964, as amended in 2008.

39. Council for International Organizations of Medical Sciences (CIOMS) and World Health Organization (WHO). International Ethical Guidelines for Biomedical Research Involving Human Subjects. Geneva: CIOMS; 2002. 


\section{The (Ab)use of Those with No Other Hope?}

40. See note 38, WMA 2008, at B12, B14-15, B18, B21; note 39, CIOMS and WHO 2002, at Guidelines $1-2,8$, and Appendix 1.

41. See note 38, WMA 2008, at B22, B24, B26-29; note 39, CIOMS and WHO 2002, at Guidelines 4-6.

42. See note 39, CIOMS and WHO 2002, at General Ethical Principles.

43. See note 38, WMA 2008, at A6, A9, B11, B21, B24.

44. See note 38, WMA 2008, at C35, emphasis supplied.

45. See note 38 , WMA 2008, at A1.

46. See note 39, CIOMS and WHO 2002, at Guideline 13.

47. See note 39, CIOMS and WHO 2002, at Commentary on Guideline 13, emphasis supplied.

48. See note 39, CIOMS and WHO 2002, at Guideline 12.

49. See note 39, CIOMS and WHO 2002, at Commentary on Guideline 12, emphasis supplied.

50. See note 39, CIOMS and WHO 2002, at Guideline 4.

51. GMC. Good Medical Practice. London: GMC; 2006, at 5.

52. See note 11, GMC 2010, at para. 4.

53. GMC. Consent: Patients and Doctors Making Decisions Together. London: GMC; 2008; GMC. Confidentiality. London: GMC; 2009.

54. See note 53, GMC 2008, at para. 9(f).

55. See note 8, RCP 2007, at para. 3.5.

56. See note $13, \mathrm{MH} 2006$, at para. 1.

57. See note 13, MH 2006, at para. 123, 132, and 134.

58. See note 13, MH 2006, at para. 134.

59. See note 13, MH 2006, at para. 135.

60. See note 12 , HSC 2003/011, at para. 1, 4, and 7.

61. See note 12, HSC 2003/011, at para. 4.

62. See note 12, HSC 2003/011, at para. 8.

63. See note 12, HSC 2003/011, at para. 10, emphasis supplied.

64. See http://www.dh.gov.uk/en/Publicationsandstatistics/Lettersandcirculars/Healthservicecirculars/ DH_4064922 (last accessed 23 Nov 2012).

65. See note 12, HSC 2003/011, at para. 2.

66. See note 12, HSC 2003/011, at para. 10.

67. DH. Health Service Circulars; http://webarchive.nationalarchives.gov.uk/20120405095111/http:// www.dh.gov.uk/en/Publicationsandstatistics/Lettersandcirculars/Healthservicecirculars/index. htm (last accessed 23 Nov 2012), emphasis supplied.

68. See note 21, NICE 2004, at 3.

69. See note 21, NICE 2004, at 3.

70. "A doctor is not guilty of negligence if he has acted in accordance with a practice accepted as proper by a responsible body of medical men skilled in that particular art": Bolam v. Friern Hospital Management Committee [1957] 2 All ER 118, 121 per McNair J.

71. Jones M. Medical Negligence. 4th ed. London: Sweet \& Maxwell; 2008, at para. 3-057.

72. See text for note 62. Competence is determined by the provisions of the Mental Capacity Act 2005.

73. Chatterton v. Gerson [1981] 1 All ER 257, 265 per Bristow J.

74. $R$ v. Brown [1994] 1 AC 212, 258-259 per Lord Mustill, HL.

75. Simms v. Simms and another; PA v. JA and another [2002] EWHC 2734.

76. See note 75, Simms, at para. 57, emphasis supplied.

77. See note 75 , Simms, at para. 57 , emphasis supplied.

78. See note 75 , Simms, at para. 48 .

79. See note 75 , Simms, at para. 48 .

80. See note 75 , Simms, at para. 48 , emphasis supplied.

81. See note 75 , Simms, at para. 49 , emphasis supplied.

82. See note 75, Simms, at para. 51, emphasis supplied.

83. See note 75, Simms, at para. 57.

84. See note 75, Simms, at para. 58, emphasis supplied.

85. See note 7, Mason, Laurie 2011, at 628.

86. DH. Xenotransplantation Guidance. London: DH; 2006, at 3.

87. See note 25, Fovargue 2012, at chap. 2. 